\title{
(PVB) FUNCTIONS AND INTEGRATION
}

\author{
D. N. SARKHEL and A. B. KAR
}

(Received 18 October 1981)

Communicated by E. Strzelecki

\begin{abstract}
We introduce the notion of functions of bounded proximal variation and the notion of orderly connected topology on the real line. Using these notions, we define in a novel way an integral of Perron type, including virtually all the known integrals of Perron and Denjoy types and admitting mean value theorems and integration by parts and the analog of Marcinkiewicz theorem for the ordinary Perron integral.
\end{abstract}

1980 Mathematics subject classification (Amer. Math. Soc.): 26 A 45, 26 A 46, 26 A 39, 26 A 03.

\section{Introduction}

In this paper we are concerned with the Lebesgue integral, the restricted Denjoy integral and its equivalent the ordinary Perron integral (see Saks (1937), Natanson (1960)), the general Denjoy integral and its equivalent the Perron integral of Ridder (1931), Section 6, the approximately continuous Perron integrals of Burkill (1931), Sonouchi and Utagawa (1949) and Kubota (1966), Section 3, and the approximately continuous Denjoy integral of Kubota (1964), which will be called here the $L-, D_{*^{-}}, P_{*^{-}}, D_{-}, P_{-}, A P_{0^{-}}, A P_{-}, A P^{*}$ - and $A D_{0^{-}}$integrals, respectively.

Considering the fact that the integrals of Perron type are defined descriptively, the most important result in the theory of the $P_{*}$-integral is the theorem of $\mathrm{J}$. Marcinkiewicz (see Saks (1937), Theorem 3.13, page 253), which states that a measurable function is necessarily $\boldsymbol{P}_{*}$-integrable on any closed interval on which it has at least one pair of continuous major and minor functions. Recently Sarkhel (1978) has proved this result under wider conditions. Unfortunately, no proper

(c) 1984 Australian Mathematical Society $0263-6115 / 84 \$ A 2.00+0.00$ 
analogs of this elegant result are known for the other integrals of Perron type. Also, the mean value theorems are not known for the approximately continuous integrals. Besides, these integrals are not all comparable. In fact, Burkill (1931), Section 1, has given a function which is $A P_{0}$-integrable but not $D$-integrable, and Tolstoff (1939), page 658, has given a function which is $D$-integrable but not $A P_{0}$-integrable. Also, the relation between the $A P$ - and $A P^{*}$-integrals is not known. We recall that the $P_{*}$-integral came as a rescue, when the $L$-integral failed to solve the problem of constructing an integral including both the integrals of Riemann and Newton.

Thus, it is expedient to seek an integral including all these integrals and admitting mean value theorems and the analog of Marcinkiewicz theorem as well. We present a complete solution to this problem, in a more general setting, by introducing a $T$-continuous Perron integral (Section 5), where $T$ is an orderly connected topology on the real line (Section 4). Our method of developing the integral has some claims to novelty and simplicity. It uses the notion of bounded proximal variation (Section 3), (PVB), which is derived from the notion of proximal absolute continuity, PAC, introduced recently by Sarkhel and De (1981), Section 5.

\section{Preliminaries}

Throughout this paper, $i, j, k, m, n$ will denote arbitrary positive integers. By a set $E$ we shall mean a subset of the real line $R,|E|$ will denote its outer Lebesgue measure, and $E^{0}$ its interior. By $f \supset E \rightarrow R[f \sim E \rightarrow R]$ we shall mean that $f$ is an extended real valued function defined and finite at least for all [almost all] points of the set $E$. For the notions of approximate continuity and derivative and of functions VB and AC we refer to Saks (1937). A function $f \supset E \rightarrow R$ is said to satisfy the condition $(N)$ of Lusin (Saks (1937), page 224) on $E$, if $|f(H)|=0$ for every $H \subset E$ with $|H|=0$.

Given $f \sim E \rightarrow R,|E|>0$, we denote by $\operatorname{ess-sup~}_{E} f\left[\operatorname{ess}^{-i n f} f_{E} f\right]$ the infimum [supremum] of the numbers $r$ such that

$$
|\{x \in E \mid f(x)>r\}|=0 \quad[|\{x \in E \mid f(x)<r\}|=0] .
$$

The function $f$ is said to be essentially bounded above [below] on $E$, if $\operatorname{ess-sup}_{E} f$ $<\infty$ [ess-inf $_{E} f>-\infty$ ].

Definition 2.1 (Sarkhel and De (1981), Section 5). A finite family (possibly empty) of pairwise disjoint open intervals with end points on a set $E$ is called a subdivision of $E$. A sequence $\left\{E_{n}\right\}$ of sets whose union is $E$ is called an $E$-form with parts $E_{n}$; if, moreover, each part $E_{n}$ is closed in $E$, then the $E$-form is said to be closed. An expanding $E$-form is called an $E$-chain. 
LEMMA 2.1. For every closed E-form $\left\{E_{n}\right\}$, there is a closed E-chain $\left\{F_{n}\right\}$ such that $F_{n}=\cup_{k \leqslant n} F_{k n}$, where $F_{k n} \subset F_{k m} \subset E_{k}$ for all $k$ and $m \geqslant n \geqslant k$, and $d\left(F_{i n}, F_{j n}\right) \geqslant 1 / n$ for $i \neq j$. (Here $d$ denotes the usual metric distance.)

Proof. Let $E_{0}=\varnothing$ and $A_{k}=\bigcup_{i \leqslant k} E_{i-1}$. For $k \leqslant n$, set

$$
F_{k n}=\left\{x \in E_{k} \mid d\left(A_{k}, x\right) \geqslant 1 / n\right\}, \quad F_{n}=\bigcup_{k \leqslant n} F_{k n} .
$$

(By definition, $d(\varnothing, x)=\infty$.) Clearly the sets $A_{k}, F_{k n}$ and $F_{n}$ are all closed in $E$, since each $E_{i-1}$ is so; also $F_{k n} \subset F_{k m} \subset E_{k}$ and $F_{n} \subset F_{n+1}$, for all $k$ and $m \geqslant n \geqslant k$. Again, for any $x \in E$, there is a unique $m$ such that $x \in E_{m} \backslash A_{m}$. Since $A_{m}$ is closed in $E$, we have $d\left(A_{m}, x\right)>0$. So $x \in F_{m n}$ for sufficiently large $n$, showing that $E=\cup_{n} F_{n}$. Finally, if $x \in F_{i n}$ and $y \in F_{j n}$ where $i<j$, then $x \in E_{i} \subset A_{j}$ and hence, by definition of $F_{j n}, d(x, y) \geqslant 1 / n$, which completes the proof.

Given $f \supset E \rightarrow R$ and $r>0$, we denote by $\underline{V}(f, E ; r)[V(f, E ; r)]$ the supremum of the sums $\Sigma\left(f\left(b_{i}\right)-f\left(a_{i}\right)\right)\left[\Sigma\left|f\left(b_{i}\right)-f\left(a_{i}\right)\right|\right]$ for all subdivisions $\left\{\left(a_{i}, b_{i}\right)\right\}$ of $E$ with $\Sigma\left(b_{i}-a_{i}\right)<r$. We define $\underline{V}(f, E ; 0)=\inf _{r>0} \underline{V}(f, E ; r), V(f, E ; 0)=$ $\inf _{r>0} V(f, E ; r)$ and $V(f, E)=\sup _{r>0} V(f, E ; r)$.

We note that $f$ is $\mathrm{VB}$ [AC] on $E$ if and only if $V(f, E)<\infty[V(f, E ; 0)=0]$. The function $f$ is said to be $\mathrm{AC}$ above [AC below] on $E$, if $\underline{V}(f, E ; 0)=0$ $[\underline{V}(-f, E ; 0)=0]$; it is (ACG) above [(ACG) below] on $E$, if it is AC above [AC below] on each part of a closed $E$-form; it is (ACG) on $E$, if it is both (ACG) above and (ACG) below on $E$ (Ridder (1931)). The terms ACG above, ACG below and ACG are defined similarly, without requiring the $E$-forms to be 'closed'. If $f$ is VB on each part of a closed $E$-form, we say that $f$ is (VBG) on $E$.

\section{3. (PVB) and (PAC) functions}

Let the functions $f, g \supset E \rightarrow R$ be given.

DEFINITION 3.1. The proximal variation of $f$ on $E$ is

$$
P V(f, E)=\inf \left\{\sup _{n} V\left(f, E_{n} ; 0\right) \mid\left\{E_{n}\right\} \text { is an } E \text {-chain }\right\} .
$$

The function $f$ is proximally variationally bounded, (PVB), on $E$, if $P V(f, E)<\infty$; it is (PAC) on $E$, if $P V(f, E)=0$.

REMARK. The properties VB through (PAC) are all necessarily hereditary. The notion of (PAC) is slightly stronger than the notion of PAC (Sarkhel and De (1981), Definition 5.1). In fact, $f$ is PAC on $E$ if and only if it is (PAC) on a co-countable subset of $E$. 
THEOREM 3.1. (i) For any two real numbers $a$ and $b$,

$$
P V(a f+b g, E) \leqslant|a| . P V(f, E)+|b| . P V(g, E) \text {. }
$$

(ii) If $P V(g, E)=0$, then $P V(f+g, E)=P V(f, E)$.

Proof. Given any real number $e>0$, there exist $E$-chains $\left\{A_{n}\right\},\left\{B_{n}\right\}$ such that $V\left(f, A_{n} ; 0\right) \leqslant P V(f, E)+e$ and $V\left(g, B_{n} ; 0\right) \leqslant P V(g, E)+e$, for all $n$. Let $E_{n}=$ $A_{n} \cap B_{n}$, then $\left\{E_{n}\right\}$ is evidently an $E$-chain. Using modulus inequalities, we readily obtain that, for each $n$,

$$
\begin{aligned}
V\left(a f+b g, E_{n} ; 0\right) & \leqslant|a| \cdot V\left(f, E_{n} ; 0\right)+|b| \cdot V\left(g, E_{n} ; 0\right) \\
& \leqslant|a| \cdot V\left(f, A_{n} ; 0\right)+|b| \cdot V\left(g, B_{n} ; 0\right) \\
& \leqslant|a| \cdot P V(f, E)+|b| \cdot P V(g, E)+e_{1},
\end{aligned}
$$

where $e_{1}=(|a|+|b|) e$. This evidently implies (i).

Next, by (i), we have

$$
\begin{aligned}
P V(f, E) & \leqslant P V(f+g, E)+P V(-g, E), \\
& \leqslant P V(f, E)+P V(g, E)+P V(-g, E),
\end{aligned}
$$

which implies (ii), since $P V(g, E)=0$ implies $P V(-g, E)=0$.

CoRollary 3.1.1. If $f$ and $g$ are both (PVB) [(PAC)] on $E$, then af + bg is also (PVB) $[(\mathrm{PAC})]$ on $E$.

Using the method of proof of Theorem 5.2 of Sarkhel and De (1981), the reader can easily verify:

THEOREM 3.2. If $|E|=0$, then $|f(E)| \leqslant P V(f, E)$.

Corollary. 3.2.1. If $f$ is (PAC) on E, then $f$ satisfies Lusin's condition $(N)$ on E.

TheOREM 3.3. Let $f$ be (PVB) on $E$. Then $f$ is (VBG) on $E$. If, further, the set $E$ is measurable, then $f \mid E$ is measurable and $f$ has a finite approximate derivative, $(a p) f^{\prime}(x)$, at almost all points $x$ of $E$.

Proof. There exist an $E$-chain $\left\{E_{n}\right\}$ and a sequence $\left\{r_{n}\right\}$ of positive numbers such that $V\left(f, E_{n} ; 2 r_{n}\right)<P V(f, E)+1$, for all $n$. Let $F_{n}$ denote the closure of $E_{n}$ in $E$. Fix an index $n$, and consider any subdivision $\left\{\left(a_{i}, b_{i}\right)\right\}$ of $F_{n}$ with $\Sigma\left(b_{i}-a_{i}\right)<2 r_{n}$. There is an index $m>n$ such that $E_{m}$ contains all the end points $a_{i}, b_{i}$. Now $E_{m} \supset E_{n}$, and, since $a_{i}, b_{i} \in F_{n}$, we can choose points of $E_{n}$ 
arbitrarily close to any point $a_{i}$ or $b_{i}$ which is not in $E_{n}$. Therefore, using modulus inequalities where necessary, we readily obtain

$$
\begin{aligned}
\sum\left|f\left(b_{i}\right)-f\left(a_{i}\right)\right| & \leqslant V\left(f, E_{n} ; 2 r_{n}\right)+2 . V\left(f, E_{m} ; 2 r_{m}\right) \\
& \leqslant 3(P V(f, E)+1)=M \quad(\text { say }) .
\end{aligned}
$$

Hence $V\left(f, F_{n} ; 2 r_{n}\right) \leqslant M$. Consequently, $f$ is VB on each of the sets $B_{n p}=F_{n} \cap$ $\left[p r_{n},(p+1) r_{n}\right]$, where $p$ is any integer (positive, negative, or zero). So $f$ is (VBG) on $E$, since the sets $B_{n p}$ determine a closed $E$-form, considering all $n$ and $p$.

When $E$ is measurable, the condition (VBG) implies that $f \mid E$ is measurable and, hence, also that $(a p) f^{\prime}=(a p)(f \mid E)^{\prime}$ exists finitely a.e. on $E$ (see Saks (1937), page 222 infra), completing the proof.

THEOREM 3.4. $P V(f, E) \leqslant \Sigma P V\left(f, E_{n}\right)$ for any closed $E$-form $\left\{E_{n}\right\}$.

Proof. Given $e>0$, for each $k$ there exist an $E_{k}$-chain $\left\{E_{k n}\right\}$ and a sequence $\left\{r_{k n}\right\}$ of positive numbers such that $V\left(f, E_{k n} ; r_{k n}\right) \leqslant P V\left(f, E_{k}\right)+2^{-k} . e$, for all $n$. Now, considering the closed $E$-chain $\left\{F_{n}\right\}$ furnished by Lemma 2.1 corresponding to the closed $E$-form $\left\{E_{n}\right\}$, and setting $H_{n}=\cup_{k \leqslant n}\left(F_{k n} \cap E_{k n}\right)$, it is easily seen that $\left\{H_{n}\right\}$ is an $E$-chain. Let $r_{n}=\min \left\{1 / n, r_{1 n}, r_{2 n}, \ldots, r_{n n}\right\}$. If $\left\{\left(a_{p}, b_{p}\right)\right\}$ is any subdivision of $H_{m}, m$ fixed, with $\Sigma\left(b_{p}-a_{p}\right)<r_{m}$, then, since $d\left(F_{i m}, F_{j m}\right) \geqslant 1 / m$ for $i \neq j$, the end points of an interval $\left(a_{p}, b_{p}\right)$ must both belong to precisely one of the sets $F_{k m} \cap E_{k m}, k=1,2, \ldots, m$, and so we clearly have

$$
\begin{aligned}
\sum_{p}\left|f\left(b_{p}\right)-f\left(a_{p}\right)\right| & \leqslant \sum_{k \leq m} V\left(f, F_{k m} \cap E_{k m} ; r_{m}\right) \\
& \leqslant \sum_{k \leq m} V\left(f, E_{k m} ; r_{k m}\right) \\
& \leqslant \sum_{k \leqslant m}\left(P V\left(f, E_{k}\right)+2^{-k} . e\right) .
\end{aligned}
$$

Hence $V\left(f, H_{m} ; r_{m}\right) \leqslant \Sigma P V\left(f, E_{n}\right)+e$, for all $m$. So $P V(f, E) \leqslant \Sigma P V\left(f, E_{n}\right)$ $+e$. Since $e>0$ is arbitrary, the proof ends.

Corollary 3.4.1. If $f$ is (PAC) on each part of a closed E-form, then $f$ is necessarily (PAC) on $E$.

Corollary 3.4.2. If $f$ and $g$ are both (PAC) on $E$, so is $\mathrm{fg}$.

Proof. By Theorem 3.3, $f$ and $g$ are both (VBG) on $E$. Clearly then $f$ and $g$ are both VB on each part $E_{n}$ of some closed $E$-form. If $M_{n}$ is a common upper bound 
of $|f|$ and $|g|$ on $E_{n}$, then for all $a, b \in E_{n}$ we have

$$
\begin{aligned}
|f(b) g(b)-f(a) g(a)| & =|g(b)(f(b)-f(a))+f(a)(g(b)-g(a))| \\
& \leqslant M_{n}(|f(b)-f(a)|+|g(b)-g(a)|) .
\end{aligned}
$$

Hence it follows at once that $f g$ is (PAC) on each of the sets $E_{n}$, which by Corollary 3.4.1 completes the proof.

COROLlaRY 3.4.3. Let $I=[a, c]$ and $J=[c, b]$; then

$$
P V(f, E \cap(I \cup J))=P V(f, E \cap I)+P V(f, E \cap J) .
$$

Proof. Given $e>0$, there exist an $E \cap(I \cup J)$-chain $\left\{E_{n}\right\}$ and a sequence $\left\{r_{n}\right\}$ of positive numbers such that $V\left(f, E_{n} ; 2 r_{n}\right) \leqslant P V(f, E \cap(I \cup J))+e$, for all $n$. Clearly then $\left\{E_{n} \cap I\right\}$ is an $E \cap I$-chain and $\left\{E_{n} \cap J\right\}$ is an $E \cap J$-chain, and $V\left(f, E_{n} \cap I ; r_{n}\right)+V\left(f, E_{n} \cap J ; r_{n}\right) \leqslant V\left(f, E_{n} ; 2 r_{n}\right) \leqslant P V(f, E \cap(I \cup J))$ $+e$, for all $n$. This implies that $P V(f, E \cap I)+P V(f, E \cap J) \leqslant P V(f, E \cap(I$ $\cup J)$ ), which by Theorem 3.4 completes the proof.

Corollary 3.4.4. Let $P V(f, E \cap[a, b])<\infty$. Then $P V(f, E \cap[a, x]) \rightarrow 0$ as $x \rightarrow a+$, and $P V(f, E \cap[x, b]) \rightarrow 0$ as $x \rightarrow b-$.

Proof. Select a strictly decreasing sequence $\left\{c_{n}\right\}$ in $(a, b)$ converging to $a$. Noting that $P V(f, E \cap\{a\})=0$, from Theorem 3.4 and Corollary 3.4.3 we readily obtain that $\sum P V\left(f, E \cap\left[c_{n+1}, c_{n}\right]\right)=P V\left(f, E \cap\left[a, c_{1}\right]\right)<\infty$, and this evidently implies that $P V(f, E \cap[a, x]) \rightarrow 0$ as $x \rightarrow a+$. Proof of the other part is similar.

THEOREM 3.5. If $f$ is (ACG) on $E$, then $f$ is (PAC) on $E$. The converse is true if $E$ is closed and $f \mid E$ is continuous.

Proof. The first part is a trivial consequence of Corollary 3.4.1. For the converse part, note that when $f$ is (PAC) on $E$, then $f$ satisfies the condition $(N)$ on $E$ by Corollary 3.2.1, and $f$ is (VBG) on $E$ by Theorem 3.3. Therefore, if it is given further that $E$ is closed and $f \mid E$ is continuous then from Banach-Zarecki theorem (Natanson (1961), page 250; Saks (1937), Theorem 6.8, page 227), it follows that $f$ is (ACG) on $E$.

THEOREM 3.6 (generalized Banach-Zarecki theorem). Let the set $E$ be closed. Then $f$ is (PAC) on $E$ if and only if $f$ satisfies the condition $(N)$ on $E$ and is (VBG) on $E$. 
Proof. The 'only if' part follows from Corollary 3.2.1 and Theorem 3.3. We prove the 'if' part in the particular case when the set $E$ is closed and bounded, and $f$ is VB on $E$ and satisfies the condition $(N)$ on $E$. The general case will then follow from Corollary 3.4.1.

Since $f$ is VB on $E, f \mid E$ is continuous n.e. on $E$. Let $d_{1}, d_{2}, \ldots$ denote the points of discontinuity, if any, of $f \mid E$. Given $e>0$, for each point $d_{n}$ we can find intervals $I_{n}=\left(p_{n}, d_{n}\right), J_{n}=\left(d_{n}, q_{n}\right)$ such that

$$
V\left(f, E \cap I_{n}\right)+V\left(f, E \cap J_{n}\right)<2^{-n} e .
$$

For each point $d_{n}$, we then fix two sequences $\left\{I_{n k}\right\}$ and $\left\{J_{n k}\right\}$ of closed intervals abutting end to end such that $\cup_{k} I_{n k}=I_{n}$ and $\cup_{k} J_{n k}=J_{n}$.

Now, the set $F=E \backslash \cup_{n}\left(I_{n} \cup J_{n}\right)$ is compact, and $f \mid F$ is continuous and VB and satisfies the condition $(N)$ on $F$. So, by Banach-Zarecki theorem, $f$ is AC on $F$. Therefore $P V(f, F)=0$. Moreover, we have

$$
\begin{aligned}
\sum_{n} \sum_{k}\left(P V\left(f, E \cap I_{n k}\right)+\right. & \left.P V\left(f, E \cap J_{n k}\right)\right) \\
& \leqslant \sum_{n} \sum_{k}\left(V\left(f, E \cap I_{n k}\right)+V\left(f, E \cap J_{n k}\right)\right) \\
& \leqslant \sum_{n}\left(V\left(f, E \cap I_{n}\right)+V\left(f, E \cap J_{n}\right)\right) \\
& <\sum_{n} 2^{-n} e=e .
\end{aligned}
$$

Hence, by Theorem $3.4, P V(f, E)<e$. Letting $e \rightarrow 0$, we get $P V(f, E)=0$. Thus $f$ is (PAC) on $E$, completing the proof.

Corollary 3.6.1. Let $\left\{p_{n}\right\}$ and $\left\{q_{n}\right\}$ denote two sequences in $R$ such that $\sum\left|p_{n}\right|<\infty$ and $q_{i} \neq q_{j}$ for $i \neq j$, and let $F: R \rightarrow R$ be defined by

$$
F(x)=\sum_{q_{n}<x} p_{n} \text { or } F(x)=\sum_{q_{n} \leqslant x} p_{n} .
$$

Then $F$ is (PAC) on $R$ and $F^{\prime}=0$ a.e. on $R$.

Proof. First suppose $p_{n} \geqslant 0$ for all $n$. Then $F$ is nondecreasing and VB on $R$. Also, if $p=\Sigma p_{n}$, then $F(R) \subset[0, p] \backslash \cup_{n}\left(F\left(q_{n}^{-}\right), F\left(q_{n}+\right)\right)$, and so we have $|F(R)|=0$, since $\Sigma\left(F\left(q_{n}+\right)-F\left(q_{n}-\right)\right)=\Sigma p_{n}=p$. Hence, by Theorem $3.6, F$ is (PAC) on $R$; also, by a known result (Varberg (1965), Theorem 12), the $L$-integral of $F^{\prime}$ on $R$ cannot exceed $|F(R)|\left(=0\right.$ ), and so $F^{\prime}=0$ a.e. on $R$ (see Natanson (1961), Theorem 6, page 138).

Since by Corollary 3.1.1 the difference of two (PAC) functions is again (PAC), the general case follows by noting that $p_{n}=\frac{1}{2}\left(\left|p_{n}\right|+p_{n}\right)-\frac{1}{2}\left(\left|p_{n}\right|-p_{n}\right)$. 
By Theorem 3.5, (ACG) functions are necessarily (PAC). We will now give an example showing how badly the converse of this result fails. This will fulfil an earlier promise (Sarkhel and De (1981), Announcement 5.1) of the first named author. We first prepare a lemma.

LEMMA 3.1. Let $G$ be an open set having right density 1 at a point $c$. Then there exist two sequences $\left\{\left[a_{n}, b_{n}\right]\right\}$ and $\left\{\left[p_{n}, q_{n}\right]\right\}$ of closed intervals such that

(i) $\cup_{n}\left[a_{n}, b_{n}\right] \subset(c, \infty) \cap G$,

(ii) $c<b_{n+1}<a_{n}<p_{n}<q_{n}<b_{n}$ for all $n$,

(iii) $\{c\} \cup \cup_{n}\left[a_{n}, b_{n}\right]$ is closed,

(iv) $\bigcup_{n}\left(p_{n}, q_{n}\right)$ has right density 1 at $c$.

ProOF. Fix a strictly decreasing sequence $\left\{c_{n}\right\}$ in $G$ converging to $c$. For each $n$, determine a finite family $\left\{\left[a_{n i}, b_{n i}\right]\right\}$ of pairwise disjoint closed intervals contained in $G \cap\left(c_{n+1}, c_{n}\right)$ such that $\Sigma_{i}\left|\left(a_{n i}, b_{n i}\right)\right|>\left|G \cap\left(c_{n+1}, c_{n}\right)\right|-$ $(1 / n)\left|\left(c_{n+2}, c_{n+1}\right)\right|$. The components of $\cup_{n} \cup_{i}\left[a_{n i}, b_{n i}\right]$ can evidently be arranged in a sequence $\left\{\left[a_{n}, b_{n}\right]\right\}$ such that $c<b_{n+1}<a_{n}$, for all $n$. For each $n$ then choose an interval $\left[p_{n}, q_{n}\right] \subset\left(a_{n}, b_{n}\right)$ such that

$$
\left|\left(a_{n}, b_{n}\right) \backslash\left(p_{n}, q_{n}\right)\right|<(1 / n)\left|\left(a_{n+1}, b_{n+1}\right)\right|
$$

These $\left\{\left[a_{n}, b_{n}\right]\right\}$ and $\left\{\left[p_{n}, q_{n}\right]\right\}$ fulfil all the requirements.

EXAMPLe 3.1. Let $P$ be a nonempty perfect set of measure 0 contained in $I=[a, b]$, with $a, b \in P$. Fix two countable dense subsets $S_{0}$ and $S_{1}$ of $P$ such that $S_{0} \cap S_{1}=\varnothing$, and such that each point of $S=S_{0} \cup S_{1}$ is a limit point of $P \backslash S$ on both sides. Let $\left\{c_{2 n-t}\right\}$ denote an enumeration (with distinct terms) of $S_{t}$, $t=0,1$. Define

$$
v(x)=\sum_{c_{n}<x}(-2)^{-n}, \quad x \in R
$$

Now, by successive applications of Lemma 3.1, we can associate with each point $c_{k}$ two sequences $\left\{\left[a_{k n}, b_{k n}\right]\right\}$ and $\left\{\left[p_{k n}, q_{k n}\right]\right\}$ of closed intervals such that:

(i) $\left|v(x)-v\left(c_{k}\right)\right|<2.2^{-k}$ for all $x \in\left(c_{k}, b_{k 1}\right)$,

(ii) $\cup_{n}\left[a_{k n}, b_{k n}\right] \subset\left(c_{k}, \infty\right) \cap I \backslash P$,

(iii) $c_{k}<b_{k m}<a_{k n}<p_{k n}<q_{k n}<b_{k n}$ for all $n, m(>n)$,

(iv) $\cup_{n}\left(p_{k n}, q_{k n}\right)$ has right density 1 at $c_{k}$,

(v) $A_{i} \cap A_{j}=\varnothing$ for $i \neq j$, where $A_{k}=\left\{c_{k}\right\} \cup \cup_{n}\left[a_{k n}, b_{k n}\right]$. 
Then, for each $k$, we define the function $F_{k}$ on $R$ by

$$
F_{k}(x)= \begin{cases}\left(v\left(c_{k}\right)-v(x)\right) \cdot \sin ^{2} \frac{\pi}{2}\left(\frac{x-a_{k n}}{p_{k n}-a_{k n}}\right) & \text { if } a_{k n} \leqslant x \leqslant p_{k n}, \\ \left(v\left(c_{k}\right)-v(x)\right) \cdot \sin ^{2} \frac{\pi}{2}\left(\frac{b_{k n}-x}{b_{k n}-q_{k n}}\right) & \text { if } q_{k n} \leqslant x \leqslant b_{k n}, \\ \left(v\left(c_{k}\right)-v(x)\right) & \text { if } x \in \cup_{n}\left(p_{k n}, q_{k n}\right), \\ 0 & \text { otherwise. }\end{cases}
$$

Consider now the function $F$ defined on $R$ by

$$
F(x)=F_{0}(x)+v(x), \quad \text { where } F_{0}(x)=\sum F_{n}(x) .
$$

By (i), the series $\Sigma F_{n}$ is uniformly and absolutely convergent. Then it is clear that $F$ is continuous everywhere except at the points $c_{k}$ on the right. But, $F(x)=v\left(c_{k}\right)$ $=F\left(c_{k}\right)$ for all $x \in \cup_{n}\left(p_{k n}, q_{k n}\right)$, which by (iv) implies that $F$ is approximately continuous at $c_{k}$ on the right. Thus $F$ is approximately continuous on $R$. Again, for each $k, F_{k}$ is evidently $\mathrm{AC}$ on $\left[a_{k n}, b_{k n}\right]$ for all $n$, and $v$ is constant on each such interval. Therefore, $F$ is (ACG) on $E=\cup_{k} \cup_{n}\left[a_{k n}, b_{k n}\right]$. Also, $F(x)=0$ for $x \leqslant a$ and $F(x)=F(b)$ for $x>b$, and $F(x)=v(x)$ for $x \in$ $I \backslash \cup_{k} \cup_{n}\left(a_{k n}, b_{k n}\right)$, and by Corollary 3.6.1 $v$ is (PAC) on $R$. Hence, by Corollary 3.4.1, $F$ is (PAC) on $R$.

Consider, on the other hand, any $(P \backslash S)$-form $\left\{P_{n}\right\}$. Since $P \backslash S$ is a $G_{\delta}$ set, there exist by Baire theorem (Saks (1937), page 54) an open interval $J$ and an index $n$ such that $J \cap P \backslash S \neq \varnothing$ and $P_{n}$ is dense in $J \cap P \backslash S$. Evidently we can find in $J$ points $c_{m}, c_{k}$ with $m$ odd, $k$ even. Since $F(x)=v(x)$ for $x \in P_{n}$, and since $v\left(c_{m}+\right)-v\left(c_{m^{-}}\right)=-2^{-m}$ and $v\left(c_{k}+\right)-v\left(c_{k}-\right)=2^{-k}$, it readily follows that $F$ is neither AC below nor AC above on $P_{n}$. Consequently, $F$ is neither ACG below nor ACG above on $P \backslash S$. So $F$ is neither ACG below nor ACG above on any superset of $P \backslash S$, not to speak of being (ACG). Noting that $I$ is a closed interval and that $F \mid(I \backslash S)$ is continuous, we see how miserably the converse part of Theorem 3.5 fails without the additional hypotheses therein.

\section{Orderly connected topology}

Let $T$ be a topology on $R$. A set $E$ will be called a $T^{+}$-vicinity $\left[T^{-}\right.$-vicinity] of a point $x$, if there is a $T$-open set $G \ni x$ such that $G \cap(x, \infty) \subset E[G \cap(-\infty, x)$ $\subset E$ ]. A set will be called $T^{+}$-open [ $T^{-}$-open], if it is a $T^{+}$-vicinity [ $T^{-}$-vicinity] of each of its points. 
Definition 4.1. Let $T_{0}$ denote the usual topology on $R$. A topology $T$ on $R$ is orderly connected, if $T \supset T_{0}$ and no interval $[a, b]$ can be expressed as the union of two disjoint sets $A, B$ such that $a \in A, b \in B, A$ is $T^{+}$-open and $B$ is $T^{-}$-open.

DEFINITION 4.2. Given two topologies $T_{1}, T_{2}$ on $R, T_{1}$ is semi-finer than $T_{2}$ at a point $x$, if every $T_{2}$-neighborhood of $x$ is either a $T_{1}^{+}$-vicinity or a $T_{1}^{-}$-vicinity of $x$.

We pause to illustrate certain aspects of our definitions. Let $T_{a}$ denote the density topology on $R$ (Goffman and Waterman (1961), Section 5). A set $G$ is $T_{a}$-open if and only if $R \backslash G$ has density 0 at each point of $G$. Approximate continuity is precisely $T_{a}$-continuity.

EXAMPle 4.1. Let $S, P, H$ denote pairwise disjoint sets such that $|S|>0$, $|P|>0,|H|=0$ and $R=S \cup P \cup H$. Let $T$ denote the family of sets $G$ such that (i) $G$ is a $T_{0}^{+}$-vicinity and a $T_{a}^{-}$-vicinity of each point of $G \cap S$, (ii) $G$ is a $T_{a}^{+}$-vicinity and a $T_{0}^{-}$-vicinity of each point of $G \cap P$ and (iii) $G$ is a $T_{0}^{+}$-vicinity and a $T_{0}^{-}$-vicinity of each point of $G \cap \mathrm{H}$. Again, let $T_{p}$ denote the family of sets $G$ such that, for every $x \in G$ and for every set $E$ having upper density $<1$ at $x,(R \backslash G) \cup E$ has upper density $<1$ at $x$. It is easily seen that $T$ and $T_{p}$ are topologies on $R$ and $T_{p} \supset T_{a} \supset T \supset T_{0}$. Now, it is known (Sarkhel and De (1981), Lemma 2.3) that, if $A \subset[a, b]$ is such that $a \in A$ and $A$ has left upper density $<1$ at each point of $B=[a, b] \backslash A$ and $B$ has right upper density $<1$ at each point of $A$, then $B=\varnothing$. From this it follows at once that $T_{p}$ is orderly connected. Since $T_{0} \subset T \subset T_{a} \subset T_{p}$, so $T_{0}, T$ and $T_{a}$ must also be orderly connected. We note further that $T_{a}$ is strictly finer than $T$, and yet $T$ is semi-finer than $T_{a}$ everywhere on $R$ or a.e. on $R$ according as $H$ is empty or not. Going through the theory of sparse sets and proximal continuity (Sarkhel and De (1981)), the reader can verify that $T_{p}$ is strictly finer than $T_{a}$ and that proximal continuity is precisely $T_{p}$-continuity.

Now let an orderly connected topology $T$ on $R$ be given. A function $f \supset[a, b]$ $\rightarrow R$ is said to have a finite right-hand T-limit $l$ at a point $c \in[a, b)$, in symbol $T f(c+)=l$, if $l \in R$ and if, for every $T_{0}$-open set $G \ni l, f^{-1}(G)$ is a $T^{+}$-vicinity of $c$. The left-hand T-limit $T f(c-)$ at a point $c \in(a, b]$ is defined analogously. Such limits are unique. To see these, it is enough to note that the intersection of two $T^{+}$-vicinities [ $T^{-}$-vicinities] of a point is again a $T^{+}$-vicinity [ $T^{-}$-vicinity] of the point, and that the empty set can neither be a $T^{+}$-vicinity nor a $T^{-}$-vicinity of any point. (If we suppose that $\varnothing$ is a $T^{+}$-vicinity [ $T^{-}$-vicinity] of a point $c$, then, since $T_{0} \subset T,[c-1, c]$ is $T^{+}$-open and $(c, c+1]$ is $T^{-}$-open $[[c-1, c)$ is 
$T^{+}$-open and $[c, c+1]$ is $T^{-}$-open], which contradicts that $T$ is orderly connected.)

The function $f$ is $T$-continuous on $[a, b]$ if and only if $T f(c+)=f(c)$ for all $c \in[a, b)$ and $T f(c-)=f(c)$ for all $c \in(a, b]$. Since $T$ is orderly connected, every subspace $([a, b], T)$ is obviously connected. So we have:

THEOREM 4.1. If $f \supset I=[a, b] \rightarrow R$ is $T$-continuous on $I$, then $f$ possesses the intermediate value property on $I$.

\section{The (TP)-integral}

Throughout this section, $T$ will denote an arbitrary but fixed orderly connected topology on $R$, semi-finer than $T_{a}$ a.e. on $R$. In $T_{0}$-topological statements, $T_{0}$ will not be mentioned.

Given $I=[a, b]$, we denote by $U(I)$ the family of functions $u \supset I \rightarrow R$ such that (i) $P V(u, I)<\infty$, (ii) $T u(x+)$ exists finitely and $u(x) \leqslant T u(x+)$, for all $x \in[a, b)$, and (iii) $T u(x-)$ exists finitely and $T u(x-) \leqslant u(x)$, for all $x \in(a, b]$. By Theorem 3.3, the condition (i) implies that (ap) $u^{\prime}$ exists finitely a.e. on $I$.

Lemma 5.1. Let $T$ be semi-finer than $T_{a}$ at all points of $I \backslash E$, where $I=[a, b]$ and $|E|=0$, and let $u \in U(I)$ be such that $(a p) u^{\prime} \geqslant 0$ on $I \backslash E$. Then $u(x)+$ $P V(u, E \cap[a, x])$ is nondecreasing on $I$.

Proof. Suppose, for a contradiction, that $u(d)+P V(u, E \cap[a, d])<u(c)+$ $P V(u, E \cap[a, c])$ for some $[c, d] \subset I$. Then, using Corollary 3.4.3, we get $0 \leqslant$ $P V(u, E \cap[c, d])<u(c)-u(d)$. Choose $e>0$ so that $P V(u, E \cap[c, d])<$ $u(c)-u(d)-e(d-c)$, and define $F(x)=u(x)+e x, x \in I$. Since $|E \cap[c, d]|$ $=0$ and $F-u$ is $\mathrm{AC}$ on $I$, using Theorems 3.2 and 3.1(ii) we get $|F(E \cap[c, d])|$ $\leqslant P V(F, E \cap[c, d])=P V(u, E \cap[c, d])<F(c)-F(d)$. Therefore, there are points $r \notin F(E \cap[c, d])$ such that $F(d)<r<F(c)$. Fix such an $r$ and set

$$
A_{0}=\{x \in[c, d] \mid F(x)>r\}, \quad B_{0}=\{x \in[c, d] \mid F(x)<r\} .
$$

Since $T_{0} \subset T$ and $u \in U(I)$, clearly $F(x) \leqslant T F(x+)$ for all $x \in[c, d)$ and $T F(x-) \leqslant F(x)$ for all $x \in(c, d]$, and so $A_{0}$ is $T^{+}$-open and $B_{0}$ is $T^{-}$-open. Again, let $F(t)=r$ for some $t \in[c, d]$. Then $t \in(c, d) \backslash E$, and so $T$ is semi-finer than $T_{a}$ at $t$ and $(a p) F^{\prime}(t)=(a p) u^{\prime}(t)+e>0$. Therefore, either $A_{0}$ is a $T^{+}$vicinity of $t$ or $B_{0}$ is a $T^{-}$-vicinity of $t$. Let $H$ denote the set of all such $t$ for which $A_{0}$ is a $T^{+}$-vicinity. Then, setting $A=A_{0} \cup H$ and $B=[c, d] \backslash A$, we see that $c \in A, d \in B, A$ is $T^{+}$-open and $B$ is $T^{-}$-open. This contradicts that $T$ is orderly connected, and the proof ends. 
Now let the functions $f, g \sim I=[a, b] \rightarrow R$ be given.

Definition 5.1. A function $u$ is a (TP)-upper function of $f$ on $I$, in symbol $u \in U(f, I)$, if $u \in U(I), u(a)=0$ and $(a p) u^{\prime} \geqslant f$ a.e. on $I$. A function $l$ is a (TP)-lower function of $f$ on $I$, in symbol $l \in L(f, I)$, if $-1 \in U(-f, I)$. For $e>0$, we set

$$
\begin{aligned}
& U_{e}(f, I)=\{u \in U(f, I) \mid P V(u, I)<e\}, \\
& L_{e}(f, I)=\{l \in L(f, I) \mid P V(l, I)<e\} .
\end{aligned}
$$

Definition 5.2. If $\lim \uparrow \inf \left\{u(b) \mid u \in U_{e}(f, I)\right\}$ and $\lim \downarrow \sup \{l(b) \mid l \in$ $\left.L_{e}(f, I)\right\}$, as $e \rightarrow 0+$, have a common finite value, then $f$ is (TP)-integrable on $I$, in symbol $f \in P(I)$, and the common limit, to be denoted by $f * I$, is the definite (TP)-integral of $f$ on $I$.

From definitions and Lemma 5.1, we readily obtain:

TheOREM 5.1. Let $u \in U(f, I)$ and $l \in L(f, I)$. Then $u-l \in U(I)$ and there exist subsets $E \subset I$, with $|E|=0$, such that $T$ is semi-finer than $T_{a}$ at all points of $I \backslash E$ and $(a p)(u-l)^{\prime} \geqslant 0$ on $I \backslash E$. For every such $E, u(x)-l(x)+P V(u-l, E$ $\cap[a, x])$ is nondecreasing and nonnegative on $I$.

In the existing theories of integrals of Perron type, the difference of an upper function and a lower function is necessarily nondecreasing. In our case, noting that $P V(u-l, E \cap[a, x])$ is nondecreasing, from Theorem 5.1 we can infer at best that $u-l$ is VB on $I$. However, using Theorems 5.1, 3.1 and noting, in particular, that $0 \leqslant u(b)-l(b)+P V(u-l, I) \leqslant u(b)-l(b)+P V(u, I)+$ $P V(l, I)$, and using accordingly minor modifications of standard arguments, there are no difficulties in proving:

THEOREM 5.2. (i) $f \in P(I)$ if and only if there exist, for every $e>0$, at least one $u \in U_{e}(f, I)$ and at least one $l \in L_{e}(f, I)$ such that $|u(b)-l(b)|<e$.

(ii) If $f \in P(I)$, then $l(b)-P V(l, I) \leqslant f * I \leqslant u(b)+P V(u, I)$, for all $l \in$ $L(f, I)$ and $u \in U(f, I)$.

(iii) If $f, g \in P(I)$ and $p, q$ are finite constants, then $(p f+q g) \in P(I)$ and $(p f+q g) * I=p \cdot(f * I)+q \cdot(g * I)$.

(iv) If $I_{1}$ and $I_{2}$ are abutting closed intervals whose union is $I$, then $f \in P(I)$ if and only if $f \in P\left(I_{1}\right) \cap P\left(I_{2}\right)$; also, when $f \in P(I)$ then $f * I=f * I_{1}+f * I_{2}$.

We see, in particular, that when $f \in P(I)$ then $f \in P([a, x])$ for all $x \in I^{0}$. We shall write $F=\operatorname{(TP})(f, I)$ to mean that $f \in P(I)$ and that $F$ is the indefinite 
(TP)-integral of $f$ on $I$, defined on $R$ by $F(x)=f *[a, x]$ for $a<x \leqslant b, F(x)=0$ for $x \leqslant a$ and $F(x)=F(b)$ for $x>b$.

Theorem 5.3. Let $F=(\mathrm{TP})(f, I)$. Then (i) $u(x)-F(x)+P V(u,[a, x])$ is nondecreasing on $I$ and $V(u-F, I) \leqslant u(b)-F(b)+2 P V(u, I)$, for all $u \in$ $U(f, I)$, and (ii) $F(x)-l(x)+P V(l,[a, x])$ is nondecreasing on $I$ and $V(F-$ $l, I) \leqslant F(b)-l(b)+2 P V(l, I)$, for all $l \in L(f, I)$.

Proof. Fix $[c, d] \subset I$. Since $u-u(c) \in U(f,[c, d])$, using Theorem 5.2(iv, ii) we get $F(d)-F(c) \leqslant u(d)-u(c)+P V(u-u(c),[c, d])=u(d)-u(c)+$ $P V(u,[a, d])-P V(u,[a, c])$, by Theorem 3.1(ii) and Corollary 3.4.3. This proves the first part of (i), from which the second part follows at once by noting that $P V(u,[a, x])$ is also nondecreasing. Proof of (ii) is similar.

TheOREM 5.4. Let $F=(\mathrm{TP})(f, I)$. Then $F$ is $T$-continuous and $(\mathrm{PAC})$ on $I$, and $(a p) F^{\prime}=$ fa.e. on $I$.

Proof. Fix $c \in[a, b)$. Given $e>0$, we can find $u \in U_{e}(f, I)$ and $l \in L_{e}(f, I)$ such that $u(b)-F(b)<e$ and $F(b)-l(b)<e$. Then, by Theorem $5.3-e<$ $u(x)-F(x)<2 e$ and $-e<F(x)-l(x)<2 e$, for all $x \in I$. Also, by definitions, $u(c) \leqslant T u(c+)$ and $T l(c+) \leqslant l(c)$, and there is a $T^{+}$-vicinity, $V$ say, of $c$ contained in $I$ such that, for all $x \in V,|u(x)-T u(c+)|<e$ and $\mid l(x)-$ $T l(c+) \mid<e$. So, for all $x \in V$, we have $F(c)-4 e<u(c)-3 e \leqslant T u(c+)-$ $3 e<u(x)-2 e<F(x)<l(x)+2 e<T l(c+)+3 e \leqslant l(c)+3 e<F(c)+4 e$, whence $|F(x)-F(c)|<4 e$. Hence $T F(c+)=F(c)$. Similarly, $T F(c-)=F(c)$ for $c \in(a, b]$. Therefore $F$ is $T$-continuous on $I$.

Next, given $e>0$, we can find $l \in L(f, I)$ such that $F(b)-l(b)+3 P V(l, I)$ $<e$. Then, by Theorems 3.1(i) and 5.3(ii), we get $P V(F, I) \leqslant P V(F-l, I)+$ $P V(l, I) \leqslant V(F-l, I)+P V(l, I)<F(b)-l(b)+3 P V(l, I)<e$. Since $e>0$ is arbitrary, it follows that $F$ is (PAC) on $I$.

Finally, by Theorem 3.3, (ap) $F^{\prime}$ exists finitely a.e. on $I$. Given $e>0$, set $I_{e}=\left\{x \in I \|(a p) F^{\prime}(x)-f(x) \mid \geqslant e\right\}$. Recalling Theorem 5.3, we can find $u \in$ $U(f, I)$ and $l \in L(f, I)$ such that $V(u-F, I)+V(F-l, I)<e^{2}$. Then, since $(a p) l^{\prime} \leqslant f \leqslant(a p) u^{\prime}$ a.e. on $I$, and since $u-F$ and $F-l$ are VB on $I$, for almost all $x \in I_{e}$ we have

$$
\begin{aligned}
e & \leqslant\left|(a p) F^{\prime}(x)-f(x)\right| \\
& \leqslant\left|(a p) u^{\prime}(x)-(a p) F^{\prime}(x)\right|+\left|(a p) F^{\prime}(x)-(a p) l^{\prime}(x)\right| \\
& =\left|(u-F)^{\prime}(x)\right|+\left|(F-l)^{\prime}(x)\right| .
\end{aligned}
$$


Also, by a known result (Varberg (1965), Theorem 14), the $L$-integral of $\left|(u-F)^{\prime}\right|$ $+\left|(F-l)^{\prime}\right|$ on $I$ cannot exceed $V(u-F, I)+V(F-l, I)$. Hence it follows that $e .\left|I_{e}\right|<e^{2}$, that is, $\left|I_{e}\right|<e$, which completes the proof, since $e>0$ is arbitrary.

It is interesting and useful to note that $F=(\mathrm{TP})(f, I)$ if and only if $F \in$ $U_{e}(f, I) \cap L_{e}(f, I)$ for every $e>0, F(x)=0$ for $x<a$ and $F(x)=F(b)$ for $x>b$. Again, Burkill and Haslam-Jones (1931) have shown that approximate derivates of measurable functions are measurable. Also, $u-F$ and $F-l$ being VB are continuous n.e. on $I$. So we readily obtain:

Corollary 5.4.1. (i) If $f \in P([a, x])$ for all $x \in I^{0}$ and if $T F(b-)$ exists finitely, where $F(x)=f *[a, x]$, then $f \in P(I)$ and $f * I=T F(b-)$.

(ii) If $f \in P([x, b])$ for all $x \in I^{0}$ and if $T F(a+)$ exists finitely, where $F(x)=f *[x, b]$, then $f \in P(I)$ and $f * I=T F(a+)$.

(iii) If $f \in P(I)$, then $f$ is measurable on I and the (TP)-upper and lower functions of $f$ on $I$ are $T$-continuous n.e. on $I$.

If $f$ is $L$-integrable on $I$, then $f \in P(I)$ since the indefinite $L$-integral is AC. On the other hand, we have:

Corollary 5.4.2. Let $F=(\mathrm{TP})(f, I), g$ be L-integrable on $I$, and $f \leqslant g$ a.e. on $I$, or, $g \leqslant f$ a.e. on I. Then $f$ is L-integrable on $I$ and $F(b)$ is the definite L-integral of $f$ on $I$.

Proof. Suppose, to fix the ideas, that $f \leqslant g$ a.e. on $I$. Let $u(x)$ denote the $L$-integral of $g$ on $[a, x], x \in I$. Clearly then $u \in U(f, I)$ and, hence, $u-F$ is VB on $I$. Since $u$ is AC, it follows that $F$ is VB on $I$, and, hence, being $T$-continuous it must also be continuous. Furthermore, being (PAC), $F$ satisfies the condition $(N)$ on $I$ by Corollary 3.2.1. Hence, by Banach-Zarecki theorem, $F$ is AC on $I$, and the proof ends by noting that $F^{\prime}=(a p) F^{\prime}=f$ a.e. on $I$.

THEOREM 5.5 (integration by parts). Let $F=(\mathrm{TP})(f, I)$ and $G=(\mathrm{TP})(g, I)$, and let $F g \in P(I)$. Then $f G \in P(I)$ and $(f G) * I=F(b) G(b)-(F g) * I$.

Proof. By Theorem 5.4, $F$ and $G$ are both $T$-continuous and (PAC) on $I$. So $F G$ is $T$-continuous on $I$ and, by Corollary 3.4 .2 , it is also (PAC) on $I$. Hence $F G=(\mathrm{TP})\left((a p)(F G)^{\prime}, I\right)$, and the proof ends by noting that $(a p)(F G)^{\prime}=f G+$ $F g$ a.e. on $I$.

The subtlety of our next two theorems is noteworthy. 
TheOREM 5.6 (first mean value theorem). Let $H=(\mathrm{TP})(f g, I), G=(\mathrm{TP})(g, I)$, $G(b) \neq 0$, and ess-inf $_{I} g \geqslant 0$, or, ess-sup $_{I} g \leqslant 0$. Set $p=H(b) / G(b)$ and $E$ $=\{x \in I \mid g(x) \neq 0\}$. Then either (i) ess-inf ${ }_{I} f<p<\operatorname{ess-sup~}_{I} f$ or, (ii) $f=p$ a.e. on $E,|E|>0$, and $p$ has one of the values ess-inf $f$ or $\operatorname{ess-sup}_{I} f .(G(b)=0$ implies $H(b)=0$.)

Proof. Assume that (i) is false. Then either $\operatorname{ess-inf}_{I}(f-p) \geqslant 0$ or $\operatorname{ess-sup}_{I}(f$ $-p) \leqslant 0$. Therefore, either $\operatorname{ess-inf}_{I}(f g-p g) \geqslant 0$ or $\operatorname{ess-sup}_{I}(f g-p g) \leqslant 0$. Hence it follows first from Corollary 5.4.2 that $(f-p) g$ is $L$-integrable on $I$, and then from the relation $(f g-p g) * I=(f g) * I-p(g * I)=H(b)-p G(b)=0$ that $(f-p) g=0$ a.e. on $I$. So $f=p$ a.e. on $E$. But $|E|>0$, since $G(b) \neq 0$. Hence ess-inf $f \leqslant p \leqslant \operatorname{ess-sup~}_{I} f$, which completes the proof, since (i) has been set aside.

Theorem 5.7 (second mean value theorem). Let $F=(\mathrm{TP})(f, I), G=(\mathrm{TP})(g, I)$, $H=(\mathrm{TP})(F g, I), G(b) \neq 0$, and ess-inf $I g \geqslant 0$, or, ess-sup $I g \leqslant 0$. Then there is $a$ point $c \in I$ such that $(f G) * I=G(b)(F(b)-F(c))$, and such that either (i) inf $f_{I} F$ $<F(c)<\sup _{I} F$, or (ii) $F=F(c)$ a.e. on $E=\{x \in I \mid g(x) \neq 0\},|E|>0$, and $F(c)$ has one of the values $\inf _{I} F$ or $\sup _{I} F$.

Proof. By Theorem 5.5, $(f G) * I=G(b)(F(b)-H(b) / G(b))$. Also, since $F$ is $T$-continuous and (PAC) on $I$, Theorem 4.1 and Corollary 3.2.1 together imply that ess-inf ${ }_{I} F=\inf _{I} F$ and ess-sup ${ }_{I} F=\sup _{I} F$. Hence the required result follows at once by using Theorems 5.6, 4.1 (with $f$ replaced by $F$ ).

Notation. For $E \subset I, f_{E}$ is the function defined on $R$ by $f_{E}(x)=f(x)$ if $x \in(\operatorname{dom} f) \cap E$ and $f_{E}(x)=0$ otherwise.

THEOREM 5.8. Let $\left\{I_{n}=\left[a_{n}, b_{n}\right]\right\}$ be a sequence of nonoverlapping closed subintervals of $I$ and $G=\cup_{n} I_{n}^{0}$. Suppose $F_{n}=(\mathrm{TP})\left(f, I_{n}\right)$ and $\Sigma_{n}\left|F_{n}\left(b_{n}\right)\right|<\infty$, and, defining $F_{0}(x)=\Sigma_{n} F_{n}(x), T F_{0}(x-)$ and $T F_{0}(x+)$ exist finitely for all $x$ and, further,

$$
\sum_{x \in R}\left\{\left|T F_{0}(x+)-F_{0}(x)\right|+\left|F_{0}(x)-T F_{0}(x-)\right|\right\}<\infty .
$$

Then $F=(\mathrm{TP})\left(f_{G}, I\right)$, where $F$ is defined on $R$ by

$$
F(x)=T F_{0}\left(x_{-}\right)-\sum_{c<x}\left(T F_{0}(c+)-T F_{0}(c-)\right) .
$$

Proof. The condition $\Sigma\left|F_{n}\left(b_{n}\right)\right|<\infty$ implies that $\Sigma F_{n}(x)$ is absolutely convergent for all $x$, and so $F_{0}$ is well defined. Again, the condition (*) implies that $F_{0}$ is $T$-continuous n.e., and that $F$ is well defined. 
Now, recalling that $T_{0} \subset T$ and using the condition (*) accordingly, it is easily seen that $T F(x+)=F(x)=T F(x-)$ for all $x$. Again, for $x \in I_{n}$ we have $F_{0}(x)=F_{0}\left(a_{n}\right)+F_{n}(x)$, which evidently implies that, for each $n, F$ is (PAC) on $I_{n}$ and $(a p) F^{\prime}=(a p) F_{n}^{\prime}=f$ a.e. on $I_{n}$. Also, for $x \in S=I \backslash G$ we have

$$
F(x)=\left\{T F_{0}(x-)-F_{0}(x)\right\}+\left\{\sum_{b_{n}<x} F_{n}\left(b_{n}\right)-\sum_{c<x}\left(T F_{0}(c+)-T F_{0}(c-)\right)\right\} .
$$

The first part on the right, being VB and 0 n.e. by the condition (*), has derivative 0 a.e., and is (PAC) on $R$, by Theorem 3.6; and the second part has also the same properties, by Corollary 3.6.1. Therefore $F$ is (PAC) on $S$ and, further, (ap) $F^{\prime}=0$ a.e. on $S$, since $G$ has density 0 a.e. on $S$.

Summing up, $F$ is $T$-continuous on $R$, it is (PAC) on $I$ by Corollary 3.4.1, and (ap) $F^{\prime}=f_{G}$ a.e. on $I$. This completes the proof, since evidently $F(x)=0$ for $x \leqslant a$ and $F(x)=F(b)$ for $x>b$.

Finally we prove the most interesting result:

THeOREM 5.9 (generalized Marcinkiewicz theorem). Suppose $f$ is measurable on I and there exist at least one $u \in U(f, I)$ and at least one $l \in L(f, I)$. Then $f \in P(I)$.

Proof. Let $E_{0}$ denote the set of the points $x \in I$ such that $f \notin P(I \cap J)$ for all closed intervals $J$ with $x \in J^{0}$. Then $E_{0}$ is closed. It is enough to show that $E_{0}=\varnothing$.

Suppose, for a contradiction, that $E_{0} \neq \varnothing$. We first show that, if $[p, q]$ is the closure of a component of $I \backslash E_{0}$, then $f \in P([p, q])$. Evidently $f \in P(J)$ for any closed interval $J \subset(p, q)$. Fix $c \in(p, q)$ and set $F(x)=f *[c, x], x \in(c, q)$. Theorem 5.3 implies that $u(x)-F(x)+P V(u,[c, x])$ and $F(x)-l(x)+$ $P V(l,[c, x])$ are nondecreasing on $(c, q)$. Since, moreover, $T u\left(q^{-}\right)$and $T l\left(q^{-}\right)$ exist finitely and $u, 1$ are (PVB) on $I$, it is readily seen that $T F(q-)$ exists finitely. So, by Corollary 5.4.1 (i), $f \in P([c, q])$. Similarly, $f \in P([p, c])$. Hence $f \in$ $P([p, q])$. In particular, therefore, $E_{0}$ is perfect.

Now, by Theorem 3.3, $u$ and $l$ are (VBG) on $I$. Then, since $E_{0}$ is perfect, using Baire theorem we can find a closed interval $J=[p, q]$, with $p, q \in E_{0}$, such that $E_{0} \cap J^{0} \neq \varnothing$ and such that $u$ and $l$ are both VB on $E=E_{0} \cap J$. Let $\left\{J_{n}=\right.$ $\left.\left[p_{n}, q_{n}\right]\right\}$ denote the sequence of the closures of the components of $J \backslash E$, and let $u_{0}$ and $l_{0}$ denote respectively the functions obtained by extending $u \mid E$ and $l \mid E$ linearly on each of the intervals $J_{n}$. Then $u_{0}$ and $l_{0}$ are both VB on $J$. Evidently $(a p) u^{\prime}=u_{0}^{\prime}$ and $(a p) l^{\prime}=l_{0}^{\prime}$, a.e. on $E$. Since, further, $(a p) u^{\prime} \geqslant f \geqslant(a p) l^{\prime}$ a.e. on $E$ and $f$ is measurable on $E$, it follows that $f_{E}$ is $L$-integrable and, hence, $f_{E} \in P(J)$. 
Next, we shall verify the hypotheses of Theorem 5.8 on $J$. As already shown, $f \in P\left(J_{n}\right)$ for all $n$. Let $F_{n}=(\mathrm{TP})\left(f, J_{n}\right)$. Theorem 5.2(ii) implies that $l\left(q_{n}\right)-$ $l\left(p_{n}\right)-P V\left(l, J_{n}\right) \leqslant F_{n}\left(q_{n}\right) \leqslant u\left(q_{n}\right)-u\left(p_{n}\right)+P V\left(u, J_{n}\right)$. Therefore $\Sigma\left|F_{n}\left(q_{n}\right)\right|<$ $\infty$, since $u$ and $l$ are VB on $E$. Hence the function $F_{0}(x)=\Sigma F_{n}(x)$ is well defined, and we have $F_{0}(x)=0$ for $x \leqslant p$ and $F_{0}(x)=F_{0}(q)$ for $x>q$.

Now fix $c \in[p, q] \backslash \cup_{n}\left[p_{n}, q_{n}\right)$. For $x \in(c, q)$ we have

$$
\begin{gathered}
F_{0}(x)=F_{0}(c)+\sum_{J_{n} \subset(c, x]} F_{n}\left(q_{n}\right) \text { if } x \in E, \\
F_{0}(x)=F_{0}(c)+\sum_{J_{n} \subset\left(c, p_{R}\right)} F_{n}\left(q_{n}\right)+F_{k}(x) \text { if } x \in\left(p_{k}, q_{k}\right) .
\end{gathered}
$$

Also, by Theorem 5.2(ii), for $x \in\left(p_{k}, q_{k}\right)$ we have

$$
\begin{aligned}
l(x)-l\left(p_{k}\right)- & P V\left(l,\left[p_{k}, x\right]\right) \\
& \leqslant F_{k}(x) \leqslant u(x)-u\left(p_{k}\right)+P V\left(u,\left[p_{k}, x\right]\right) .
\end{aligned}
$$

Moreover, since $u-1$ is VB on $I$, and since $c$ is a limit point of $E$ on the right, we have $T u(c+)-T l(c+)=T(u-l)(c+)=(u-l)(c+)=\left(u_{0}-l_{0}\right)(c+)$ $=u_{0}(c+)-l_{0}(c+)$, whence

$$
T u(c+)-u_{0}(c+)=T l(c+)-l_{0}(c+) .
$$

Finally, when $q_{k} \rightarrow c+$ in (3), then $l\left(p_{k}\right)=l_{0}\left(p_{k}\right) \rightarrow l_{0}(c+)$ and $u\left(p_{k}\right)=$ $u_{0}\left(p_{k}\right) \rightarrow u_{0}(c+)$, and, by Corollary 3.4.4, $P V\left(l,\left[p_{k}, x\right]\right) \rightarrow 0$ and $P V\left(u,\left[p_{k}, x\right]\right) \rightarrow 0$.

It is now clear from (2) through (4) that, if $J \backslash E$ is a $T^{+}$-vicinity of $c$, then $T F_{0}(c+)=F_{0}(c)+T u(c+)-u_{0}(c+)$. Since $u(c)=u_{0}(c)$, this relation can be written as

$$
T F_{0}(c+)-F_{0}(c)=(T u(c+)-u(c))-\left(u_{0}(c+)-u_{0}(c)\right) .
$$

If, on the other hand, $J \backslash E$ is not a $T^{+}$-vicinity of $c$, then every $T^{+}$-vicinity of $c$ intersects $E$, implying thereby that $u_{0}(c+)=T u(c+)$ and $l_{0}(c+)=$ $T l(c+)$; consequently then it follows from (1) through (4) that $T F_{0}(c+)=F_{0}(c)$.

Again, for all $x \in \cup_{n}\left[p_{n}, q_{n}\right)$ we clearly have $T F_{0}(x+)=F_{0}(x)$. Thus, $T F_{0}(x+)$ exists for all $x \in R$ and, further,

$$
\left|T F_{0}(x+)-F_{0}(x)\right| \leqslant|T u(x+)-u(x)|+\left|u_{0}(x+)-u_{0}(x)\right| .
$$

Similarly, $T F_{0}(x-)$ exists for all $x \in R$ and, further,

$$
\left|F_{0}(x)-T F_{0}(x-)\right| \leqslant|u(x)-T u(x-)|+\left|u_{0}(x)-u_{0}(x-)\right| \text {. }
$$

But, by definitions of $u, l$ we have $0 \leqslant T u(x+)-u(x) \leqslant T u(x+)-u(x)+$ $l(x)-T l(x+)=T(u-l)(x+)-(u-l)(x)=(u-l)(x+)-(u-l)(x)$, and similarly, $0 \leqslant u(x)-T u(x-) \leqslant(u-l)(x)-(u-l)(x-)$. Therefore, recalling that $u-l$ and $u_{0}$ are both VB on $J$, from (5) and (6) we conclude that 
$\Sigma_{x \in R}\left|T F_{0}(x+)-F_{0}(x)\right|<\infty$ and $\Sigma_{x \in R}\left|F_{0}(x)-T F_{0}(x-)\right|<\infty$. Hence, by Theorem 5.8, $f_{G} \in P(J)$ where $G=\cup_{n} J_{n}^{0}$. Since, moreover, $f_{E} \in P(J)$ and $f_{J}=f_{E}+f_{G}$, it follows that $f \in P(J)$. This contradicts that $E_{0} \cap J^{0} \neq \varnothing$, and the proof ends.

\section{Relation with other integrals}

The inclusion relations among the known integrals are as follows: $L \subset P_{*}=$ $D_{*} \subset P=D \subset A P^{*}=A D_{0}$ (Kubota (1966), Theorem 3.6), $A P_{0} \subset A D_{0}$ (Kubota (1964), Theorem 2) and $A P_{0} \subset A P$ (Sonouchi and Utagawa (1949), Definition 1.1). In our case, by Example 4.1, $\left(T_{a} P\right) \subset\left(T_{p} P\right)$.

Now, if $f$ is $A D_{0}$-integrable on $I$, then the corresponding indefinite integral $F$ is $T_{a}$-continuous and (ACG) (and, hence, (PAC) by our Theorem 3.5) on $I$, and $(a p) F^{\prime}=f$ a.e. on $I$. Hence $F=\left(T_{a} P\right)(f, I)$, proving that $A D_{0} \subset\left(T_{a} P\right)$.

Next, let $f$ be $A P$-integrable on $I$, and let $F$ be the corresponding indefinite integral. Then $F$ is $T_{a}$-continuous on $I$ and $(a p) F^{\prime}=f$ a.e. on $I$. Also, given $e>0$, there exists $A P$-upper and lower functions $u, l$ such that $u(b)-F(b)<e$ and $F(b)-l(b)<e$; besides, $u-F$ and $F-l$ are nondecreasing on $I$ and, by definition, the lower approximate derivates of $u(=F+(u-F))$ and $-1(=-F$ $+(F-l)$ ) are greater than $-\infty$ at all points of $I$. Then, following the proof of Theorem 5.4(ii) of Sarkhel and De (1981), we can find $I$-chains $\left\{E_{n}\right\}$ and $\left\{F_{n}\right\}$ such that $\underline{V}\left(-F, E_{n} ; 0\right)<e$ and $\underline{V}\left(F, F_{n} ; 0\right)<e$, for each $n$. Now $\left\{E_{n} \cap F_{n}\right\}$ is an $I$-chain and, using Lemma 5.2(i) of Sarkhel and De (1981), we have, for each $n, V\left(F, E_{n} \cap F_{n} ; 0\right) \leqslant \underline{V}\left(F, E_{n} \cap F_{n} ; 0\right)+\underline{V}\left(-F, E_{n} \cap F_{n} ; 0\right) \leqslant \underline{V}\left(F, F_{n} ; 0\right)+$ $\underline{V}\left(-F, E_{n} ; 0\right)<2 e$. Hence it follows that $F$ is (PAC) on $I$. Consequently $F=$ $\left(T_{a} P\right)(f, I)$, proving that $A P \subset\left(T_{a} P\right)$. Thus our contemplation is fulfilled.

Finally, we note that the function $F$ of Example 3.1 is neither ACG below nor ACG above on $I$, and yet $F=\left(T_{a} P\right)\left((a p) F^{\prime}, I\right)$.

\section{References}

J. C. Burkill (1931), 'The approximately continuous Perron integral,' Math. Z. 34, 270-278.

J. C. Burkill and U. S. Haslam-Jones (1931), 'The derivates and approximate derivates of measurable functions,' Proc. London Math. Soc. (2) 32, 346-355.

C. Goffman and D. Waterman (1961), 'Approximately continuous transformations,' Proc. Amer. Math. Soc. 12, 116-121.

Y. Kubota (1964), 'An integral of the Denjoy type,' Proc. Japan Acad. 40, 713-717.

Y. Kubota (1966), 'An integral of the Denjoy type. II,' Proc. Japan Acad. 42, 737-742. 
I. P. Natanson (1961), Theory of functions of a real variable, vol. I (rev. ed., Ungar, New York).

I. P. Natanson (1960), Theory of functions of a real variable, vol. II (Ungar, New York).

J. Ridder (1931), 'Über den Perronschen Integralbegriff und seine Beziehung zu den $R$-, $L$ - und D-integralen,' Math. Z. 34, 234-269.

S. Saks (1937), Theory of the integral (2nd rev. ed., PWN, Warsaw).

D. N. Sarkhel (1978), 'A criterion for Perron integrability,' Proc. Amer. Math. Soc. 71, 109-112.

D. N. Sarkhel and A. K. De (1981), 'The proximally continuous integrals,' J. Austral. Math. Soc. Ser. A 31, 26-45.

G. Sonouchi and M. Utagawa (1949), 'The generalized Perron integrals,' Tôhoku Math. J. 1, 95-99.

G. Tolstoff (1939), 'Sur l'intégrable de Perron,' Recueil Math. 47, 647-659.

D. E. Varberg (1965), 'On absolutely continuous functions,' Amer. Math. Monthly 72, 831-841.

\section{Department of Mathematics}

University of Kalyani

Kalyani, West Bengal

India 